\title{
Biotechnologies et brevets : vers la normalisation
}

Les progrès de l'ingéniérie génétique amènent les industriels à concevoir et à créer de nouvelles espèces vivantes : avant tout, pour l'instant, des micro-organismes génétiquement recombinés. Le problème se pose des moyens de la protection de ces «inventions» d'un type tout nouveau et donc de la nature des brevets auxquels elles peuvent donner lieu.

\section{Ernest Gutmann}

\section{Conseil en brevets d'invention}

\section{RÉFÉRENCES}

1. Rote Taube (pigeon rouge). IIC 1970 ; 1: 136 .

2. In re Diamond V. Chakrabarty. USPQ $1980 ; 193: 206$.

\section{ADRESSE}

E. Gutmann : 67 boulevard Haussmann, 75008 Paris, France.

\section{TIRÉS A PART}

E. Gutmann : 67 boulevard Haussmann, 75008 Paris, France.

$\mathrm{m} / \mathrm{s} \mathrm{n}^{\circ} 4$ vol. 4 , avril 88 es biotechniques ou, comme il est désormais convenu de les appeler, les «biotechnologies » font aujourd'hui figure de science nouvelle, dont les applications industrielles à venir suscitent dans l'opinion publique tout à la fois des appréhensions et des espoirs extrêmes. Qu'ils soient ou non fondés, une autre question a rapidement été posée, celle de la protection éventuelle des produits de ces recherches et des moyens mis en œuvre pour les obtenir, en l'occurrence des organismes vivants, micro-organismes et même organismes supérieurs, le cas échéant transformés par l'homme.

\section{Brevetabilité des micro- organismes modifiés}

La brevetabilité possible de micro-organismes modifiés par l'homme est d'ores et déjà acquise dans la plupart des grands pays industriels du monde occidental, même si les conditions auxquelles leur protection est subordonnée ne sont pas toujours satisfaisantes.

La validité de brevets dans ce domaine dépend souvent du dépôt des souches des micro-organismes concernés auprès de Col- lections habilitées*, lorsqu'il peut être jugé que le public n'avait pas auparavant accès à ces microorganismes ou que ceux-ci ne pouvaient pas être décrits dans le brevet de façon qu'un homme du métier puisse ensuite exécuter l'invention. Ces Collections habilitées sont alors généralement tenues de délivrer ces souches aux tiers qui en font la demande. Dans certains cas cet acte équivaut à une remise de «l'usine clefs en mains » du breveté à ses concurrents, sans qu'il soit possible dans la pratique, pour le premier, d'exercer un contrôle sur l'usage que les seconds font ensuite de ses souches.

Notre propos n'est pas une tentative d'imaginer de nouvelles formes de protection pour les inventions appartenant au domaine des biotechnologies, compte tenu de leurs spécificités propres. Il serait plutôt une tentative de

\footnotetext{
* Les Collections habilitées sont des organismes privés ou semi-privés, officiellemen reconnus par des Offices de Brevets comme étant aptes, contre le versement d'une rémunération par les demandeurs, à recevoir des cultures de micro-organismes, aux fins de procédures de délivrance de brevets. Ces Collections s'obligent à mettre ces cultures de microorganismes à la disposition des tiers dans des conditions expressément prévues par des textes ayant force de loi à l'égard de ces demandes de brevets ou brevets, après leur publication.
} 
démonstration que les biotechnologies, pour autant qu'on en discerne les évolutions futures, sont au contraire appelées à « rentrer dans le rang » et qu'à l'instar de la pratique pour d'autres inventions, leur protection devrait être acquise sur la base de leur seule description dans les brevets devant les protéger.

Ces questions méritent d'autant plus d'être posées que les problèmes soulevés par la protection de ces techniques ont beaucoup évolué. Pour tenter d'y voir plus clair, un bref retour en arrière peut être utile.

C'est un truisme de dire que les «biotechnologies» remontent en fait à la plus haute antiquité car leur utilisation pour la production des denrées alimentaires essentielles est on ne peut plus ancienne. Plus près de nous, les fermentations représentent depuis longtemps une part non négligeable des activités des industries chimiques et pharmaceutiques. La culture de micro-organismes nouveaux et leur amélioration par mutagenèse a permis de sélectionner des souches hyperproductives de diverses substances (acides aminés, vitamines, antibiotiques, etc.) dans des milieux et des conditions données. Avant l'ère du génie génétique, la jurisprudence avait déjà consacré la brevetabilité de ces nouveaux procédés microbiologiques et des produits nouveaux en résultant.

En revanche, les micro-organismes eux-mêmes, en tant qu'entité vivante, sont longtemps restés exclus de la brevetabilité. Cette exclusion ne semble d'ailleurs pas avoir trop entravé le développement de cette industrie, encore récemment plus lent que pour d'autres. C'est ce que l'on serait tenté de croire, par exemple à la lumière de l'affirmation que l'on trouve encore dans un article publié sur les «fermentations» (Encyclopedia Universalis, 1976, vol. 6), selon laquelle les techniciens du domaine définissaient les fermentations comme «tout processus au cours duquel est utilisé un micro-organisme spécifique pour produire en culture..., une substance chimique définie, cette substance chimique ne pouvant être synthétisée par voie chimique ou cette synthèse étant d'un prix de revient trop élevé ».

Il n'est pas sûr que les nouvelles techniques, et parmi elles le génie génétique, aient déjà à ce jour surmonté ces difficultés, notamment en termes de coût de production. En revanche, elles donnent d'ores et déjà accès à des produits qui ne pouvaient être auparavant obtenus qu'en quantités infinitésimales impropres à toute exploitation industrielle: insuline humaine, urokinase, activateur du plasminogène tissulaire, hormone de croissance, pour ne citer que quelques exemples. Elles permettent grâce au transfert d'une information génétique déterminée d'une espèce vivante à une autre, totalement étrangère à la première, de créer des organismes cellulaire dotés de caractéristiques nouvelles identifiables, leur conférant la capacité de synthétiser des produits que les mêmes organismes, non transformés, n'avaient nullement vocation naturelle à produire. A titre d'exemple, on mentionnera l'incorporation, dans des cellules en culture provenant d'un hôte notoirement insensible au virus de l'hépatite $B$, d'une partie de l'information génétique portée dans le génome de ce dernier, permettant désormais à ces cellules de produire des principes immunogènes vaccinants contre cette maladie. Est-il besoin de souligner que la maîtrise de l'information génétique, portée par des acides nucléiques, clonés ou synthétisés, ouvre donc des perspectives sans limites en ce qui concerne la production, par des organismes vivants spécialement adaptés à cet effet, de molécules totalement nouvelles, dont la structure peut être entièrement «dictée» par l'homme. Tels sont, à n'en pas douter, certains des buts du génie génétique ou de «l'ingéniérie génétique », équivalent exact de l'expression anglaise genetic engineering, qui rend bien compte de la nature des techniques en cause.

\section{Un nouveau type de brevet?}

La nouveauté ou plutôt le caractère singulier des biotechnologies, tel qu'il est généralement appréhendé par le public, se situe à plusieurs niveaux: défi que représente la capacité acquise par l'homme de transformer la matière vivante et de franchir des barrières jusqu'alors «biologiquement $\gg$ insurmontables entre espèces hétérologues, potentialités importantes de ces techniques encore inconcevables au début des années 1970, accélération considérable des recherches dans ce domaine et concurrence déjà acharnée entre les plus grandes firmes industrielles, notamment pharmaceutiques.

Cela explique la remise en cause des moyens de protection des inventions relevant des biotechnologies et les études qui sont menées par les cercles interessés, notamment l'Organisation Mondiale de la Propriété Intellectuelle (OMPI) qui a désigné à cet effet un comité d'experts. La question est donc clairement posée : le système des brevets peut-il désormais pourvoir à la protection de toutes les formes d'inventions de la biotechnologie, et ce dans des conditions semblables à celles prévalant dans d'autres domaines techniques ou fautil, à leur intention, créer de nouveaux systèmes de protection?

Sans doute faut-il, avant de proposer une réponse éventuelle à cette question - en tout cas au niveau de techniques mettant en œuvre des organismes unicellulaires - , rappeler quelques-uns des principes sur lesquels tous les droits de brevets sont fondés. Ils découlent de la conviction partagée par toutes les sociétés, que le progrès technique est directement lié à la rapidité avec laquelle les inventions sont rendues publiques par leurs auteurs. Les droits de brevets ont donc d'abord pour but d'y inciter et le droit temporaire exclusif d'exploitation de l'invention qu'ils confèrent ne représente en faitque la contrepartie offerte à celui qui, le premier, 
en fait la description dans un titre réservé à cet effet : le brevet.

Le brevet a donc une double fonction. La première fonction du brevet, on l'a vu, est de décrire l'invention, de façon telle qu'elle puisse être effectivement et librement exécutée par les tiers, après expiration du droit exclusif temporaire d'abord réservé à l'inventeur. L'autre fonction du brevet est de définir l'étendue du droit que l'inventeur veut et peut se réserver, au vu de l'état connu de la technique. La définition, la délimitation de la protection demandée, font l'objet des revendications du brevet.

Pour que cette double fonction puisse réellement être effectuée, il faut que l'invention soit d'une nature telle qu'elle puisse être à la fois décrite et définie.

\section{Breveter des}

\section{organismes supérieurs?}

On comprendra mieux, à la lumière de ce qui précède, les raisons pour lesquelles la doctrine a longtemps admis, même en dehors de toute considération éthique, quelquefois encore opposée aujourd'hui à la brevetabilite d'inventions portant sur la matière vivante, que les inventions du domaine du vivant ne pouvaient pas, en général, être à la fois décrites et définies comme les objets inertes. Cette non-brevetabilité allait tellement de soi qu'elle n'a longtemps fait l'objet d'aucune disposition particulière dans le cadre des droits de brevets.

Un arrêt célèbre de la Cour Suprême allemande, rendu dans l'affaire dite du «pigeon rouge ${ }^{*}[1]$ a néanmoins, dès 1969 , jeté un premier doute sérieux sur le bien-fondé de l'exclusion de principe de toute matière vivante de la brevetabilité. La Cour Suprême allemande avait été saisie d'un appel d'une juridiction inférieure rejetant une demande de brevet revendiquant ce curieux animal. Elle devait en définitive conclure à l'absence de validité du brevet litigieux, car la description du brevet ne garantissait pas que le procédé d'obtention de pigeons $m / s n^{\circ} 4$ vol. 4 , avril 88 rouges pût être reproduit. Exclusion de la brevetabilité et impossibilité de décrire des procédes de fabrication «d'inventions vivantes » paraissaient encore en 1969 - intimement liées. Mais la Cour Suprême allemande indiquait aussi que le défaut de validité du brevet tenait à ce motif particulier, et non au principe qu'un animal nouveau pût faire l'objet d'un brevet, puisqu'à l'époque la loi allemande en vigueur n'excluait pas expressément les races animales de la brevetabilité.

De fait, la situation allait rapidement évoluer et le principe de la brevetabilité possible de la matière vivante allait être consacré par une décision, retentissante à l'époque, de la Cour Suprême américaine d a n s l' a f f a i re «in re Chakrabarty** [2], selon laquelle l'absence de dispositions dans la loi américaine interdisant la brevetabilité d'inventions ressortissant au domaine du vivant n'autorisait pas les instances judiciaires à conclure à leur défaut de brevetabilité, dès lors qu'elles étaient le résultat d'une intervention de l'homme sur la matière. La Cour Suprême américaine devait donc conclure à la brevetabilité d'une souche bactérienne, à laquelle avaient été incorporés plusieurs plasmides capables d'induire la production par cette souche d'enzymes permettant de dégrader des hydrocarbures, notamment pour la destruction des «marées noires ».

L'introduction effective dans des droits de brevets de dispositions relatives au sort fait à certaines inventions se rattachant au do-

* Décision de la Cour Suprême Fédérale de la RFA du 27 mars 1969 dans l'affaire dite du "pigeon rouge". Cette décision a fait l'objet d'une publication en anglais dans une revue connue sous la désignation "IIC Studies" (abréviation IIC) et publiée par le "MaxPlanck institute for foreign and international patent, copyright and competition law, Munich, $R F A$ ».

** "in re" : expression dérivée du latin, suivie du nom de l'une des parties et utilisée aux Etats-Unis pour faire référence à un cas de Etats-Unis pour faire référence a un cas de
jurisprudence, en l'occurrence la décision de la Cour Suprême des États-Unis du 16 juin 1980 dans l'affaire Diamond v. Chakrabarty, publiée dans une revue trimestrielle dite "United States Patents Quaterly", connue sous l'abréviation USPQ. maine du vivant est en fait un événement très récent. De ce point de vue, l'article 53 b de la convention sur le brevet européen (CBE), était une innovation en soi, même si sa rédaction découlait d'une convention antérieure signée à Strasbourg en 1963 appelant à l'harmonisation des droits de brevet en Europe. En effet l'article $53 \mathrm{~b}$ de la $\mathrm{CBE}$, dont l'esprit se retrouve dans l'article 7, paragraphe c, de la loi française du 2 janvier 1968 modifiée par la loi du 13 juillet 1978, dispose que: "Les brevets européens ne sont pas délivrés pour... b) les variétés végétales ou les races animales ainsi que les procédés essentiellement biologiques d'obtention de végétaux ou d'animaux, cette disposition ne s'appliquant pas aux procédés microbiologiques et aux produits obtenus par ces procédés."

Nous n'entrerons pas dans une exégèse des inventions assimilables à des variations végétales ou à des races animales qui sont exclues de la brevetabilité par la Convention Européenne. Rappelons néanmoins brièvement que les variétés végétales nouvelles peuvent, dans de nombreux pays, faire l'objet de titres de protection distincts des brevets, les certificats d'obtention végétale, et que l'une des raisons sous-tendant leur exclusion de la brevetabilité trouve son origine dans la Convention Internationale pour la protection des obtentions végétales, signée à Paris le 2 décembre 1961. Celle-ci interdit le cumul des protections des variétés végétales simultanément par des brevets et des certificats d'obtention végétale. Quant aux races animales, la brevetabilité (qui pour certains milieux intéressés devrait être admise) pose de sérieux problèmes éthiques. Elle pourrait aussi se heurter, au moins en partie, à une autre exclusion de la brevetabilité découlant de l'article 52 (4) de la CBE, excluant de toute protection « les méthodes de traitement chirurgical ou thérapeutique du corps humain ou animal». Une telle exclusion deviendrait opérante pour une invention «biotechnologique » 
ayant vocation à pallier une déficience génétique chez l'homme ou l'animal.

En revanche l'article 53 (b) consacre la brevetabilité possible des procédés microbiologiques, donc des procédés du génie génétique visant à la transformation de micro-organismes ou de cultures cellulaires pour les rendre aptes à exécuter des fonctions qu'elles ne pouvaient naturellement exercer. Les produits obtenus par ces procédés microbiologiques ne sont pas davantage exclus par principe de la brevetabilité et il était clair dès l'origine que ces produits «non vivants» (par exemple: produits issus de fermentations classiques) pouvaient être protégés. A ces derniers s'ajoute «l'outillage » des biotechnologies modernes, par exemple des ADNs ou fragments d'ADNs purifiés, des ADNs recombinants divers, des plasmides, des anticorps monoclonaux, produits parfaitement inertes, lorsqu'ils ne sont pas placés dans un environnement leur permettant d'exercer leurs propriétés spécifiques.

Qu'en était-il des «produits vivants » auxquels conduisaient les procédés microbiologiques non exclus de la brevetabilité ? La question, comme nous venons de le voir, a longtemps été controversée. Il convient de rendre cette justice à l'Office Européen des Brevets qui avait dès l'origine (avant la décision « in re Chakrabarty ») accepté que ces produits vivants, non spécifiquement exclus de la brevetabilité puissent être revendiqués dans un brevet européen, dès lors qu'ils satisfaisaient aux critères de brevetabilité usuels (possibilité d'application industrielle, nouveauté et activité inventive). La brevetabilité de micro-organismes ou de cellules en culture transformées par de nouveaux procédés biotechnologiques n'est plus aujourd'hui sérieusement mise en cause.

\section{Protéger l'invention biotechnologique}

La différence principale subsistant encore à ce jour entre les inventions relevant des biotechnologies industrielles - mettant en jeu des cultures de cellules et les autres inventions réside dans l'obligation de déposer auprès de collections habilitées les micro-organismes mis en jeu dans les inventions, dès lors qu'ils ne sont pas accessibles au public ou qu'ils ne peuvent pas être décrits dans la demande de brevet européen, de façon à permettre à l'homme du métier d'exécuter l'invention. Les dispositions réglementaires que l'on retrouve maintenant dans de nombreux droits de brevets mettent la preuve de l'accessibilité possible des tiers aux micro-organismes ou cultures cellulaires concernées à la charge du demandeur du brevet, s'il n'a pas effectué le dépôt auprès d'une Collection habilitée, alors que pour d'autres inventions, la preuve qu'elles ne peuvent pas être exécutées par l'homme du métier doit être rapportée par les tiers.

Cette situation défavorable aux inventeurs du domaine qui nous préoccupe prend un relief particulier quand on sait que de nombreuses collections ont été habilitées à recevoir des dépôts de souches dans le cadre de procédures de brevets. Pourtant, s'il est une caractéristique nouvelle que l'on peut dégager des biotechnologies modernes, c'est qu'à ce jour les caractéristiques imprimées à la matière vivante pour la rendre capable d'exécuter de nouvelles fonctions peuvent de plus en plus souvent être à la fois décrites et définies. Le plus souvent, la capacité acquise par des cultures cellulaires déterminées à exercer de nouvelles fonctions s'analyse à partir de l'information génétique qui leur a été incorporée au moyen de procédés microbiologiques ou génétiques. Or cette information génétique est fondée sur la façon dont sont assemblés les uns aux autres les quatre nucléotides essentiels intervenant dans la constitution des acides nucléiques et formés à partir de quatre bases: adénine, cytosine, guanine et thymine.

Il est d'ores et déjà possible dans de nombreux cas, et cela sera encore plus fréquent à l'avenir, de corréler exactement les fonctions acquises par des micro-organismes, aux modifications structurales induites par des procédés reproductibles parfaitement accessibles à la description. Les progrès rapides des techniques de synthèse chimique des acides nucléiques, selon le programme qu'ils doivent exécuter au sein de l'hôte cellulaire dans lequel ils sont ensuite introduits d'une part, et de techniques de séquençage d'ADN, d'autre part, ne peuvent que s'accompagner de la disparition du caractère aléatoire des mutations que l'on s'efforçait auparavant d'induire dans les cultures cellulaires utilisées dans les fermentations classiques. De ce fait, il n'est pas douteux que les inventions biotechnologiques sont appelées à rejoindre les autres inventions, dès lors que celles-ci deviennent, comme celles-là, accessibles à la description et à la définition dans des demandes de brevet.

\section{Conclusion}

Plus rien ne s'oppose à ce que le brevet reste donc l'instrument de choix pour protéger les inventions du domaine des biotechnologies. Le système des brevets repose sur l'utilisation du langage, le meilleur outil de communication que l'on puisse concevoir entre l'inventeur et le public. Comment pourrait-on mieux à la fois décrire et définir l'invention, si ce n'est en ayant recours aux ressources du langage. Les biotechnologies n'échappent pas aux lois qui régissent les autres activités de recherche. Leur développement suppose des investissements considérables. Ils ne sont sérieusement envisagés par ceux qui les consacrent à la recherche biotechnologique que s'ils peuvent espérer une rémunération raisonnable de leurs investissements. Aucun système de protection autre que le brevet n'a à ce jour démontré sa capacité à le remplacer efficacement à de telles fins 


\section{Summary}

Fermentation processes, accordingly biotechnological techniques, have been used in the antiquity and ever since. However inventions in this art long relied mostly on screening procedures or contingent mutations in existing microorganisms, hence have long been excluded from patentability on the ground that they were not acessible to description in such manner as to enable their reproduction. However recent case law, and thereupon patent laws and implementing regulations have provided for the deposition of microorganisms with officially recognized depositories substantially as a substitute for their description. Most striking aspects of modern biotechnology lie with the mastering by man of the transfer of genetic information from one live species to another, coupled with the capacity conferred on the latter to produce new products or to operate new functions correlated with that transfer. The nucleic acids which bear that information can be identified, hence become amenable to description and definition. Biotechnological inventions should thus become protectable by patents in as efficient a manner as other inventions bearing on non-live matter.

\section{ERRATA}

- En page 141, du no 3, vol. 4, une erreur s'est glissée dans l'Introduction de l'article de J. Chelly: le poids moléculaire de la dystrophine est de 400000 et non pas de 40000 .

- En page 150 du même article, dans les remerciements, c'est J. Chelly qui est boursier de l'AFM et non le Pr. J.-C. Kaplan.

$m / s n^{\circ} 4$ vol. 4, avril 88 